\title{
Research on plastic waste treatment
}

\author{
Baolin $\mathrm{Ye}^{*}$ \\ School of Electrical and Electronic Engineering, North China Electric Power University, Baoding \\ 071003, Hebei, China
}

\begin{abstract}
Plastics are widely used in our daily lives, and our lives cannot do without plastics. This paper introduces the concepts of relative irreplaceable coefficient and absolute irreplaceable coefficient, discusses the extent to which plastic waste can be reduced to an environmentally safe level, and reversely applies a health risk assessment model to describe the environmental safety level, and compares this safety level as a warning line for the amount of plastic waste.
\end{abstract}

Keywords: Plastic Waste, Replacement, Substitution Coefficient, Environmental Safety Evaluation Model.

\section{Introduction}

\subsection{Background}

A world without plastics seems un-imaginable today [1]. While plastic products provide convenience to people's production and life, they also lead to an increasing number of plastic wastes, that is, serious plastic pollution problems. With the intensification of pollution problems, how to reduce the generation of plastic waste and improve management measures have become the focus of attention. These plastic wastes are often made of chemical raw materials such as polyvinyl chloride (PVC), polyethylene (PE), polypropylene (PP), and polystyrene (PS). Since plastics are difficult to degrade in the natural environment, they will cause great harm to the environment. Scattered waste can hinder the beauty and cleanliness of the city; large amounts of agricultural film remaining in farmland can easily cause soil compaction and crop production; scattered waste that is randomly discarded Easily eaten by animals[2].Therefore, there is an urgent need for a model to estimate the maximum discharge of plastic waste under the premise of environmental safety. It is hoped that this will become the scientific basis for adopting phased measures to reduce the amount of global plastic waste. 


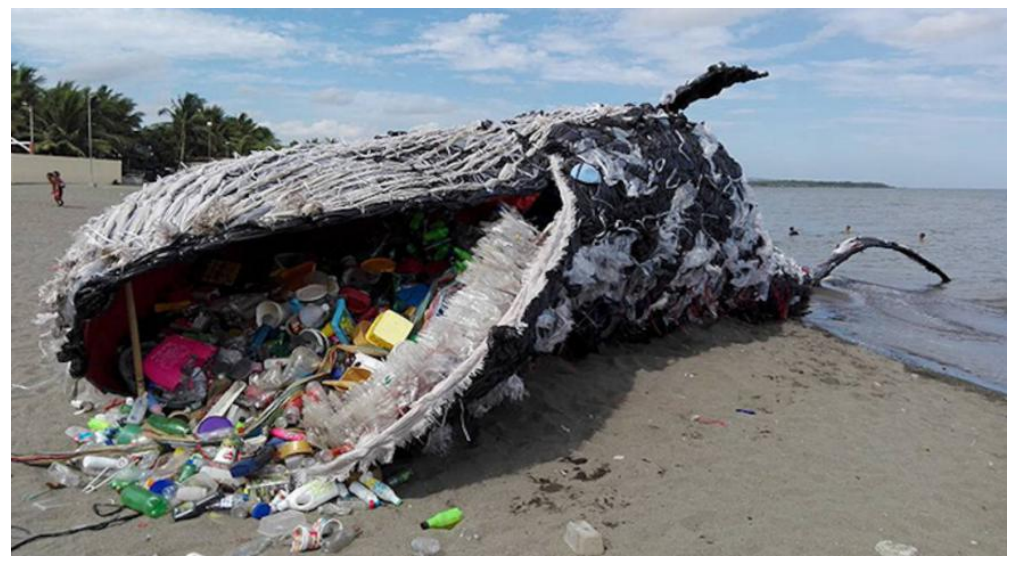

Figure 1 A whale made of plastic waste and stuffed with plastic waste

\subsection{Literature review}

\subsubsection{Concept of substitution coefficient}

Here we introduce the concepts of absolute irreplaceability and relative irreplaceability. Based on the impact of these plastic products on people's daily lives, we define:

$$
\begin{aligned}
& \text { relative irreplaceable coefficient }=\frac{\text { Current degree of Plastic replacement }}{\text { Degree of Plastic replacement without action }} \\
& \text { absolutely irreplaceable coefficient }=\frac{\text { Essential degree of Plastic replacement }}{\text { Degree of Plastic replacement without action }}
\end{aligned}
$$

After introducing the relative irreplaceable coefficient and the absolute irreplaceable coefficient, we can use it to describe the replaceability of plastic products. If the absolute irreplaceable coefficient of a plastic product is 0 , we consider that such plastic product can be completely replaced.

Due to changes in population and economy over time, the relative and absolute irreplaceable coefficients are not static. Moreover, it is clear that the relative irreplaceable coefficient $\leq$ absolute irreplaceable coefficient

\section{Alternative Analysis of Plastic Products}

There are countless types of plastic products. Among them, products with short life cycle are more likely to become the source of plastic waste due to its nearly $100 \%$ discard rate. Such single-use plastic products are packaging bags, agricultural mulch, disposable tableware, plastic bottles which are selected as research object here. At the same time, due to their high discard rate, for the sake of discussion we assume that the output of these plastic products is equal to the amount of plastic waste generated.

When considering the minimum level of plastic waste that can be reduced in a certain region, country, and continent, we can instead consider the reducing the minimum level of plastic products used in that region. The plastic products here are only analyzed for the above four single-use plastic products. 


\subsection{Application and Alternative Analysis of Plastic Products}

Absolute irreplaceability and relative irreplaceability can be used to quantitatively describe the degree of substitution of plastic products.

a) Plastic packaging bag is widely used in people's daily life, generally for package food and daily necessities, and often discarded after people unpack. The main substitutes for plastic bags are wrapping paper, cotton bags or cotton bags, Stainless steel or glass containers, bamboo or straw baskets, or even long-term plastic containers, which can largely replace the use of plastic packaging bags in daily life. Its absolute irreplaceable coefficient of plastic packaging bag $\leq 10 \%$

b) Agricultural mulch is a plastic film used in agricultural production. As an important means of production of modern agricultural development, the application of agricultural mulch has greatly promoted the increase of agricultural yield and efficiency. Because of its irreplaceable role in agricultural production, there are no substitutes that can replace agricultural mulch film on a large scale. Therefore, the absolute irreplaceable coefficient of agricultural mulch $\geq 90 \%$

c) Disposable tableware is widely used in the catering industry, it was created to improve the convenience of dining. However, its convenience does not mean that it cannot be replaced, and traditional tableware and tableware can even bring a better dining experience than disposable tableware. We think its absolute irreplaceable constant $\leq 5 \%$.

d) Plastic bottle. There are a variety of alternatives to plastic bottles, such as stainless-steel bottles and cartons, and the market share of plastic bottles is not overwhelming. We think its absolute irreplaceable constant $\leq 6 \%$

\subsection{Factors affecting the relative irreplaceable coefficient}

\section{- Civic life}

Plastic products, such as plastic packaging bags are widely used in citizen life because of their convenience and low price. Therefore, plastic products will be difficult to replace because of citizens' existing needs and dependence on them. This demand and dependence will also prevent the reduction in the use of plastics, which is manifested in the suppression of reducing the relatively irreplaceable coefficient

\section{- Urban, regional, national and continental policies}

To maintain environmental safety, regions will formulate a series of policies to compulsively reduce the use of plastic products. The promulgation of these policies will promote the reduction of the Plastic replacement, which correspond to the reduction relatively irreplaceable coefficient. In developing countries, the restrictions on plastics have weakened its low-price advantage to a certain extent so that citizens' demand for plastic bags has decreased.

\section{- Socioeconomic}

Due to the huge demand for plastic products in the society, the plastic industry has become an indispensable part of the national manufacturing industry and made a huge contribution to the Socioeconomic. Limiting the production of plastic means a huge blow to the industry, and even have a huge impact on society. Therefore, it will prevent the reduction of plastic replacement, also showing as suppressing the reduction of the relatively irreplaceable coefficient.

\section{- Environmental conditions}

The environment is the foundation of people's survival. Plastic pollution will cause great damage to the environment. When the environmental conditions are extreme, people will not be able to survive. Therefore, the harsh environmental conditions will promote the replacement of plastic products, which is reflected in the reduction of the relatively 
irreplaceable coefficient.

\section{The Design of Environmental Safety Evaluation Model}

The US Environmental Protection Agency has proposed a health risk assessment model[3]. This model uses the degree of risk as an evaluation index to link environmental pollution with human health, quantitatively describe the risk of pollutants causing harm to human health and has certain applications throughout the world[4,5]. By modifying this model, we established an Environmental Safety Evaluation model (ESE), which can be mainly divided into four parts: Hazard Identification, Risk Characterization, Does-Response Assessment and Exposure Assessment.

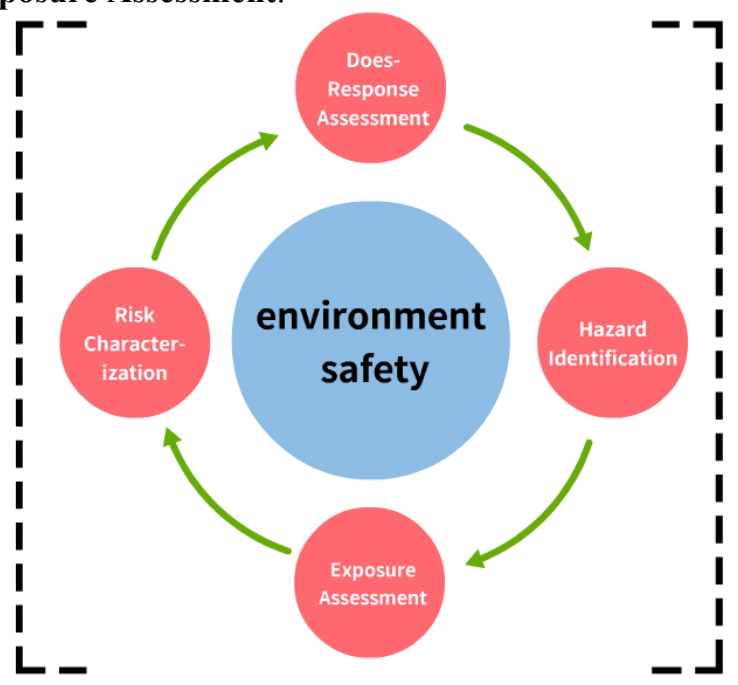

Figure 2 Framework of Environmental Safety Evaluation model

\subsection{Hazard Identification}

The core of this part is to answer two questions: Is there evidence that the pollutants pose a health hazard to the exposed population and which chemicals are harmful to the exposed population.

First, we identify the harmful substances that may be present in plastic waste. The white pollution mainly refers to the environmental pollution caused by the disposal of plastic products such as plastic bags, disposable tableware, and agricultural mulch made of high molecular compounds such as polyethylene, polypropylene, and polyvinyl chloride. Therefore, in this discussion, we will mainly use recycled polyethylene plastic bags as examples.

The harmful substances contained in recycled polyethylene plastic bags mainly include the following

- Heavy metal harmful substances: including lead $(\mathrm{Pb})$, chromium $(\mathrm{Cd})$, antimony $(\mathrm{Sb})$ and arsenic (As) in pigments and dyes.

- Organic and harmful substances: including residual organic solvents in plastic bags

\subsection{Risk Characterization}

Risk characterization determine the probability of a risk occurring by comprehensive toxicity 
assessment and exposure assessment.

For carcinogens, it is generally considered that there is no risk threshold, that is, as long as they exist, it will adversely affect the human body. Therefore, we cannot quantitatively determine the maximum exposure level of a carcinogen by limiting the carcinogenic risk.

For non-carcinogenic risk (conventional toxicity risk), it is generally believed that there is a measurement threshold for the response of organisms to non-carcinogens. Below this threshold there is no adverse effect on health. When calculating multiple substances, the non-carcinogenic risk of each substance is generally calculated, and then add them up without considering synergy and antagonism. Non-carcinogenic risk is usually described by the risk index (HI), which is defined as the ratio of the long-term daily intake dose to the reference dose due to exposure.

$$
H I=\frac{\text { Long-term daily dose }}{\text { Reference dose }}=\left\{\begin{array}{lr}
>1, & \text { high threat to environmental organisms } \\
\leq 1, & \text { No threat to environmental organisms }
\end{array}\right.
$$

The total value of $H I$ cannot be greater than 1 , otherwise it will pose a threat to living things. Therefore, the HI of each harmful non-carcinogenic substance must not exceed $1 / k$, where $k$ is the number of harmful species in the pollutant.

\subsection{Does-Response Assessment}

Does-Response Assessment is an assessment of the correlation between a person's exposure to pollutants and the possibility of negative effects. It provides toxicity data for risk characterization, including carcinogenic and non-carcinogenic effects of harmful substances.

In the original model, the possibility of a certain hazard in the population was analyzed and judged through comprehensive toxicity evaluation and exposure evaluation. Here we reversely apply the original model. On the premise that the toxicity evaluation data is a fixed value, the maximum exposure level of the human body to a certain harmful substance can be obtained by limiting the possibility of a certain hazard in the population.

The US Environmental Protection Agency (EPA) classifies hazardous substances into the following 5 categories [6].

Table 1 Carcinogen classification

\begin{tabular}{|c|l|l|}
\hline \multirow{4}{*}{ Carcinogen } & Type A & \multicolumn{1}{c|}{ Human carcinogens } \\
\cline { 2 - 3 } & \multirow{2}{*}{ Type B } & $\begin{array}{l}\text { Suspected human carcinogens } \\
\text { B1: Limited carcinogenicity data } \\
\text { B2: There is only sufficient evidence } \\
\text { of carcinogenicity to animals }\end{array}$ \\
\hline \multirow{2}{*}{ Non-carcinog } & Type C & Potential human carcinogens \\
\cline { 2 - 3 } & Type D & Non-carcinogen \\
\cline { 2 - 3 } & Type E & Well-confirmed non-carcinogen \\
\hline
\end{tabular}

What needs to be clear is that carcinogens also cause non-carcinogenic risks. Non-carcinogenic risks mean conventional toxicity risks rather than no toxicity risks.

The required non-carcinogenic reference dose (RFD) of the substance is found in the US IRIS (Integrated Risk Information System) and other references Exposure Assessment. 
Table 2 Hazardous Substances toxicity data[7]

\begin{tabular}{|c|c|c|}
\hline $\begin{array}{c}\text { Harmful } \\
\text { Substance }\end{array}$ & $\begin{array}{c}\text { Carcinogenicity } \\
\text { Determination }\end{array}$ & Non-carcinogenic Reference of Dose $\left(\mathrm{mg} \mathrm{kg}^{-1} \mathrm{~d}^{-1}\right)$ \\
\hline $\mathrm{Cr}$ VI & D & $3.00 \mathrm{E}-03$ \\
\hline $\mathrm{As}$ & $\mathrm{A}$ & $3.00 \mathrm{E}-04$ \\
\hline $\mathrm{Cd}$ & $\mathrm{B} 1$ & $5.00 \mathrm{E}-04$ (Water) \\
& - & $4.00 \mathrm{E}-03$ (Food) \\
\hline $\mathrm{Sb}$ & $\mathrm{B} 2$ & $4.00 \mathrm{E}-04$ \\
\hline $\mathrm{Pb}$ & - & $1.40 \mathrm{E}-04$ \\
\hline Toluene & - & $8.00 \mathrm{E}-02$ \\
\hline
\end{tabular}

\subsection{Exposure Assessment}

The exposure assessment mainly studies the amount of exposure, the frequency of exposure, the intensity of exposure, the route of exposure, and the duration of exposure. Here we first analyze the exposure pathways of harmful substances entering the human body, and then quantitatively calculate the amount of harmful substances entering the human body through this pathway [7].

The original model used the experimental data to quantitatively calculate the exposure of harmful substances entering the organism through various exposure pathways. Here we reversely apply the original model to determine the highest level of human exposure to a harmful substance.

\subsubsection{Exposure method}

In general, the main routes of exposure are oral (ingestion), nasal (inhalation), direct skin contact, etc. Since harmful substances enter the body mainly through the enrichment effect of the food chain, the exposure route under this discussion is mainly oral intake.

\subsubsection{CDI calculation of long-term daily intake}

According to the US Environmental Protection Agency (USEPA) recommendation method, it is calculated by the following formula:

$$
C D I=R F D * H I
$$

Where $C D I$ is the long-term daily intake due to exposure, $\mathrm{mg} /(\mathrm{kg} * \mathrm{~d})$;

$R F D$ is the reference dose of the hazardous substance found in the Does-Response Assessment, $\mathrm{mg} /(\mathrm{kg} * \mathrm{~d})$;

$H I$ is a non-carcinogenic risk index obtained during the Risk Characterization.

\subsubsection{Calculation of maximum intake}

According to the US Environmental Protection Agency (USEPA) recommended method, the oral route exposure level is calculated by the following formula.

$$
C D I_{\text {oral }}=C W * I R * E F * E D * B W^{-1} * A T^{-1}
$$

In the formula, $C D I_{\text {oral }}$ is the chronic daily intake; $I R$ is the intake (assuming that adults consume about $1 \mathrm{~L}$ of contaminated food per day, the $I R$ value should be $1 \mathrm{~L} /$ day); $E F$ Is exposure frequency (value 365); $E D$ is exposure period (value 30 for non-carcinogen); 
$B W$ is the body weight; $A T$ is average time (value 10950 for non-carcinogen); $C W$ is chemical concentration in water The average enrichment ratio of the food chain is generally 10 times.

The length of the food chain where people live is generally level 4. Therefore $C W * 10^{4}$ is characterized by the content of harmful substances in contaminated food that adults consume daily.

\subsection{Analysis of Uncertainty Factors in ESE Model}

One of the basic characteristics of environmental risk assessment is uncertainty [5]. In addition to plastic waste itself generating toxic and harmful substances, many research reports also show that plastic debris may absorb water chemical pollutants and spread to higher levels through the food chain. Therefore, under real conditions, a local survey of the amount of hazardous substances in plastic waste should be followed to re-draft the risk standard.

\section{Conclusion}

Affected by factors such as plastic waste disposal space, temperature, and local population density, it is difficult to make a quantitative determination from the level of plastic waste in the environment to the environmental safety. Here we use the local water composition as the total indicator to characterize the level of environmental safety. We will continue to reduce the amount of plastic waste in the environment to meet the safe standards when the concentration of harmful substances in the water composition is unqualified.

The level of environmental safety is used as a standard of the success on reducing the level of plastic waste. It cannot influence the substitution degree of plastic products. Because the evaluation of environmental safety level is based on the effects of harmful substances on organisms or the environment while the calculation of the substitution factor is based on needs of human society and the limitations of industrial technology. But the former can be used as a reference line to continuously show the distance to the environmental safety alert line for each region. Thus, the appropriate policies and management solutions can be adopted.

\section{References}

1. Geyer, R., Jambeck, J. R., \& Law, K. L. (2017). Production, use, and fate of all plastics ever.

2. Xudong Song, Analysis of the treatment and reuse of plastic waste[J].Journal of Meteorology and environment.2006 (05)

3. Risk Assessment Guidance for Superfund, Vol. I. Human Health Evaluation Manual. USEPA. EPA/540/1-89/002. 1989

4. Zeng Guangming, Zhuo Li, Zhong Zhenglin, Zhang Panyue, Li Xinmin. Water environment health risk assessment model and its application [J]. Hydropower Energy Science. 1997 (04)

5. Mao Xiaoling, Liu Yangsheng. Research Progress on Environmental Risk Assessment at Home and Abroad [J]. Journal of Applied Basics and Engineering Science. 2003 (03)

6. Integrated Risk Information System (Electronic data base). USEPA. http://www.epa.Gov/iris . 2009 
7. Oak Ridge Reservation Annual Site Environmental Report for 2005. Oak Ridge National Laboratory. http //www.ornl.gov/aser. 2006 